\title{
Whale optimization algorithm with nonlinear control parameter
}

\author{
Minghui Zhong ${ }^{1}$, and Wen Long ${ }^{2 *}$ \\ ${ }^{1}$ College of Information Engineering, Guangxi University of Foreign Language, Nanning 530222, PR China \\ ${ }^{2}$ Key Laboratory of Economics System Simulation, Guizhou University of Finance \& Economics, Guiyang 550025, PR China
}

\begin{abstract}
Whale optimization algorithm (WOA) is a relatively novel intelligence optimization technique which has been shown to be competitive to other population-based algorithms. However, the control parameter $a$ is a major factor to affect the algorithm's convergence precision and speed. At present, few of them are aiming at control parameter setting in WOA algorithm. This paper proposes corresponding improved WOA algorithm with different nonlinear adjustment strategy of control parameter $a$ by adopting sinusoid, cosine, tangential, logarithmic and quadratic curves. The experimental results for six benchmark test functions show that the proposed nonlinear adjustment strategies are superior to the classical linear strategy.
\end{abstract}

Keywords: whale optimization algorithm; control parameter; nonlinear; benchmark test function

\section{Introduction}

Whale optimization algorithm (WOA) is a relatively new meta-heuristic optimization technique proposed by Mirjalili and Lewis [1], which inspires the bubble-net hunting behaviour of humpback whales. WOA easy to implement and has few adjustment parameters, which make it superior than particle swarm optimization (PSO), grey wolf optimizer (GWO) algorithm, and gravitational search algorithm (GSA), etc. As a result, WOA has attracted much attention and has been applied to handle many practical engineering application problems, such as training multi-layer perceptron in neural network [2], tracking MPP of photovoltaic system [3], optimizing the active and reactive power dispatch problem [4], feature selection [5], parameter estimation of photovoltaic cells [6], multilevel thresholding image segmentation [7], and so on.

Similarly to other meta-heuristic algorithm, as the growth of the search space dimension, WOA is also easily trapped in the local optimum and provides a poor convergence [8]. Consequently, researchers increasingly are paying close attention to improve the performance of WOA. For instance, Trivedi et al. [8] proposed an improved version of WOA (called AWOA) based on modified position-updated equation. Ling et al. [9] developed an improved version of WOA (called LWOA) with Levy flight trajectory to make faster convergence and avoid premature convergence. $\mathrm{Hu}$ et al. [10] presented an improved version of WOA (called IWOA) based on inertia weight.

In the standard WOA, the distance control parameter $a$ is an important parameter to balance the abilities of exploration and exploitation. However, the distance control parameter $a$ is linearly decreased from 2 to 0 over the course of iterations [1]. Thus, in this paper, we introduce the different distance control parameter strategies to WOA to design the improved whale optimization algorithms (IWOAs). The proposed IWOAs are tested with six benchmark functions and the results show that the proposed algorithm is effective in most cases. The structure of the rest of this paper is as follows. In Section 2, the standard WOA is summarized. The improved version of WOA is proposed in Section 3. In Section 4, six benchmark functions are introduced to test the performance of proposed algorithm. Finally, Section 5 summarizes the conclusion of this paper.

\section{Whale optimization algorithm}

Fig. 1 shows the bubble-net hunting behaviour of humpback whales. Humpback whales prefer to hunt school of krill or small fishes close to the surface. Humpback whales swim around the prey within a shrinking circle and along a spiral-shaped path simultaneously to create distinctive bubbles along a circle or "9"-shaped path [1]

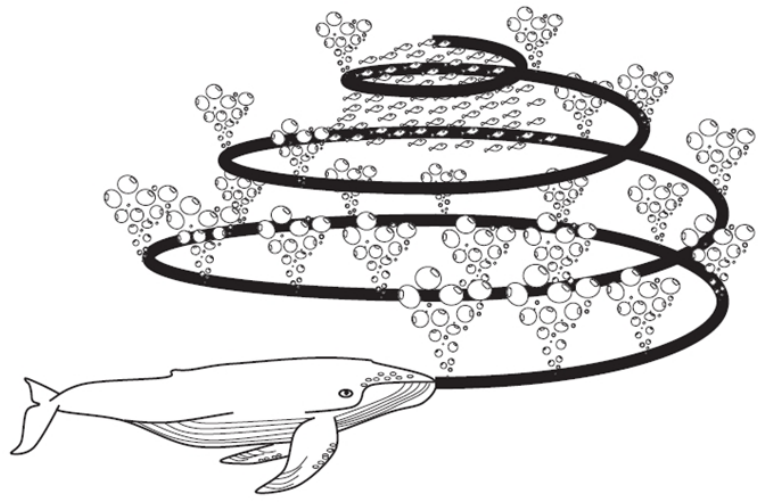

Figure 1. Bubble-net feeding behaviour of humpback whales.

In 2016, Mirjalili and Lewis [1] developed a novel meta-heuristic optimization algorithm, WOA, which mimics the bubble-net hunting technique of humpback whales. To simulate this technique in WOA, there is a probability of $50 \%$ to choose between the shrinking encircling mechanism and the spiral model to update the position of whales during optimization.

\footnotetext{
* Corresponding author: 1w227@mail.gufe.edu.cn
} 


\subsection{Shrinking encircling prey}

In WOA, to mathematically model the encircling mechanism, the following formulas are introduced [1]:

$$
\begin{gathered}
\stackrel{\sqcup}{X}(t+1)=\stackrel{\sqcup}{X}^{*}(t)-A \cdot\left|C \cdot \stackrel{\sqcup}{X}^{*}(t)-\stackrel{\sqcup}{X}(t)\right| \\
A=2 \cdot a \cdot r-a \\
C=2 \cdot r
\end{gathered}
$$

$\stackrel{\text { X }}{X}$ is the position vector, $\stackrel{\mathrm{U}}{X}^{*}$ is the historically best position, $t$ indicates the current iteration, $r$ is a random number in $[0,1], a$ is called distance control parameter and is linearly decreased from 2 to 0 over the course of iterations [1]:

$$
a(t)=2-2 \frac{t}{t_{\max }}
$$

where $t_{\max }$ indicates the maximum number of iterations.

\subsection{Spiral bubble-net feeding strategy}

A spiral equation is used between the position of whale and prey to mimic the helix-shaped movement of humpback whales as follows:

$$
\stackrel{\sqcup}{X}(t+1)=\stackrel{\sqcup}{X}^{*}(t)+e^{b l} \cdot \cos (2 \pi l) \cdot\left|\stackrel{\sqcup}{X}^{*}(t)-\stackrel{\sqcup}{X}(t)\right|
$$

where $b$ is a constant for defining the shape of the logarithmic spiral and $l$ is a random number in $[-1,1]$.

\subsection{Search of prey}

In order to have a global optimizer, when $A$ is greater than 1 or less than -1 , the search agent is update according to a randomly chosen search agent instead of the best search agent:

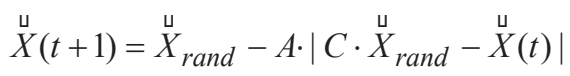

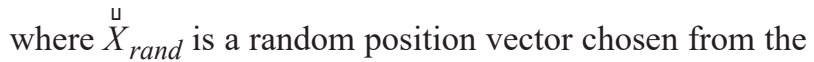
current population.

For further details, the reader may refer to [1].

\section{Improved WOA algorithm}

As we know, meta-heuristic optimization algorithm must has a good balance between exploration and exploitation. From [1] and Eq.(1), exploration and exploitation abilities of WOA are guaranteed by the adaptive values of distance control parameter $a$. But the values of distance control parameter $a$ are linearly decreased from 2 to 0 over the course of iterations.

Although the linearly distance control parameter strategy shows faster convergence and high accuracy in the early stage for most cases by empirical investigations with some well-known problems [10]. However, the actual search process of WOA is nonlinear and much more complicated, so it can not be truly reflected by the linearly decreasing adaptive parameter $a$. Therefore, inspired by PSO algorithm, this work proposes some improved versions of WOA by introducing non-linear strategies of distance control parameter $a$ :

$$
\begin{gathered}
a(t)=\left(a_{\text {max }}-a_{\text {min }}\right) \times \sin \left(\mu \cdot \frac{t}{t_{\text {max }}} \pi\right) \\
a(t)=\left(a_{\text {max }}-a_{\text {min }}\right) \times \cos \left(\mu \cdot \frac{t}{t_{\text {max }}} \pi\right) \\
a(t)=\left(a_{\text {max }}-a_{\text {min }}\right) \times \tan \left(\mu \cdot \frac{t}{t_{\text {max }}} \pi\right) \\
a(t)=\left(a_{\text {max }}-a_{\text {min }}\right) \times \log \left(0.5+(e-1) \times\left(\frac{t}{t_{\text {max }}}\right)\right) \\
a(t)=\left(a_{\text {max }}-a_{\text {min }}\right) \times\left(\frac{t}{t_{\text {max }}}\right)^{2}
\end{gathered}
$$

where $t$ indicates the current iteration, $t_{\max }$ indicates the maximum number of iterations, $\mu$ is the nonlinearly adjust factor, $a_{\max }$ and $a_{\min }$ are the maximum and minimum values of the distance control parameter $a$, respectively.

Based on the above considerations, the flow chart of improved version of WOA is showed in Fig.2.

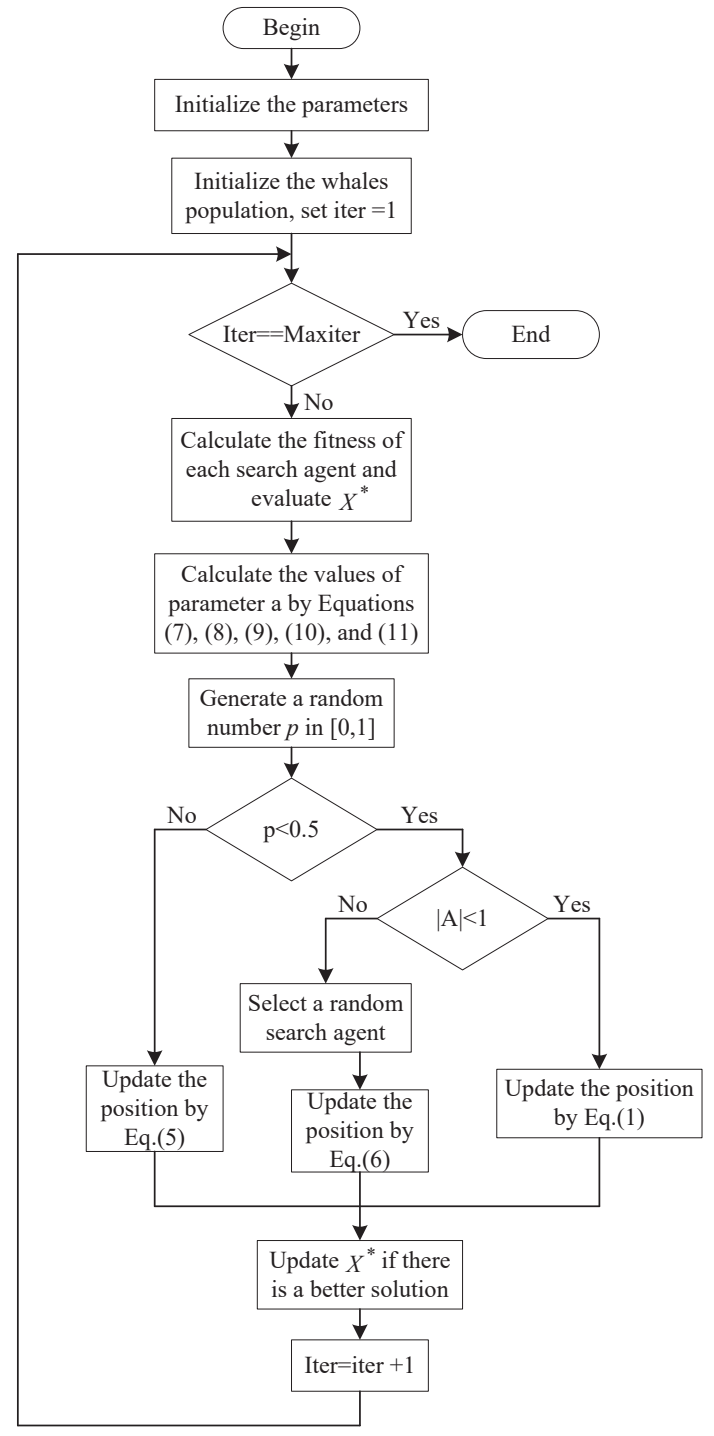

Figure 2. The flow chart of improved WOA algorithm. 
Thus four kinds of improved WOAs are obtained as follows:

(1) WOA with nonlinear distance control parameter strategy shown Eq.(7) (donated as SinWOA).

(2) WOA with nonlinear distance control parameter strategy shown Eq.(8) (called as CosWOA).

(3) WOA with nonlinear distance control parameter strategy shown Eq.(9) (called as TanWOA).

(4) WOA with nonlinear distance control parameter strategy shown Eq.(10) (called as LogWOA).

(5) WOA with nonlinear distance control parameter strategy shown Eq.(11) (called as SquareWOA).

\section{Simulation and comparison}

To test the performance of the proposed algorithm, we select six benchmark functions from literature [1]. These functions are summarized in Table 1 .

Table 1. Benchmark test functions.

\begin{tabular}{|l|l|}
\hline Function & Search range \\
\hline$f_{1}(x)=\sum_{i=1}^{n} x_{i}^{2}$ & {$[-100,100]$} \\
$f_{2}(x)=\sum_{i=1}^{n}\left|x_{i}\right|+\prod_{i=1}^{n}\left|x_{i}\right|$ & {$[-10,10]$}
\end{tabular}

$$
\begin{array}{l|l}
f_{3}(x)=\sum_{i=1}^{n}\left(\left\lfloor x_{i}+0.5\right\rfloor\right)^{2} & {[-100,100]} \\
f_{4}(x)=\sum_{i=1}^{n} i x_{i}^{4}+\operatorname{random}[0,1) & {[-1.28,1.28]} \\
f_{5}(x)=\sum_{i=1}^{d}\left[x_{i}^{2}-10 \cos \left(2 \pi x_{i}\right)+10\right] & {[-5.12,5.12]} \\
f_{6}(x)=\frac{1}{4000} \sum_{i=1}^{d} x_{i}^{2}-\prod_{i=1}^{d} \cos \left(\frac{x_{i}}{\sqrt{i}}\right)+1 & {[-600,600]}
\end{array}
$$

The set of experiments test on six functions are executed. For a fair comparison among six algorithms, they are tested using the same setting of the parameters, that is, the population size is equal to 30 , the maximum iteration is equal to 500 , and the function dimension is set to 30 . The other parameters of our algorithm are set as follows: $a_{\max }=2, a_{\min }=0, \mu=7$. Table 2 shows the best result (Best), the mean result (Mean), and the standard deviation (Std) result. All source codes are written in MATLAB 7.11.0 (win64) and executed in an Apple iMac computer with $2.8 \mathrm{GHz}$ Intel Core i5, 8 GB 1867 $\mathrm{MHz}$ DDR3 under MAC OS operation system. All results reported are obtained based on 30 independent runs. For clarity, the results of the best algorithms are marked in boldface.

Table 2. Benchmark test functions.

\begin{tabular}{|c|c|c|c|c|c|c|c|}
\hline Function & Result & WOA & SinWOA & CosWOA & TanWOA & LogWOA & SquareWOA \\
\hline & Best & $2.94 \mathrm{E}-083$ & $4.67 \mathrm{E}-112$ & $\mathbf{3 . 6 4 E}-\mathbf{1 2 8}$ & $5.15 \mathrm{E}-115$ & $5.62 \mathrm{E}-113$ & $7.62 \mathrm{E}-091$ \\
\hline$f_{1}$ & Mean & $5.51 \mathrm{E}-075$ & $4.34 \mathrm{E}-100$ & $\mathbf{2 . 3 8 E}-\mathbf{1 2 2}$ & $3.18 \mathrm{E}-108$ & $4.46 \mathrm{E}-102$ & $1.49 \mathrm{E}-082$ \\
\hline & Std & $1.23 \mathrm{E}-076$ & $1.94 \mathrm{E}-100$ & $\mathbf{4 . 1 0 E}-\mathbf{1 2 2}$ & $5.00 \mathrm{E}-108$ & $3.10 \mathrm{E}-102$ & $1.29 \mathrm{E}-082$ \\
\hline & Best & $3.97 \mathrm{E}-056$ & $1.15 \mathrm{E}-075$ & $\mathbf{3 . 2 3 E}-\mathbf{0 7 7}$ & $2.05 \mathrm{E}-076$ & $4.41 \mathrm{E}-070$ & $1.95 \mathrm{E}-062$ \\
\hline$f_{2}$ & Mean & $6.42 \mathrm{E}-053$ & $2.64 \mathrm{E}-069$ & $\mathbf{2 . 5 1 E}-\mathbf{0 7 3}$ & $3.58 \mathrm{E}-071$ & $1.63 \mathrm{E}-066$ & $1.43 \mathrm{E}-059$ \\
\hline & Std & $8.65 \mathrm{E}-053$ & $7.36 \mathrm{E}-069$ & $\mathbf{3 . 4 5 E}-\mathbf{0 7 3}$ & $7.52 \mathrm{E}-071$ & $1.19 \mathrm{E}-065$ & $1.09 \mathrm{E}-058$ \\
\hline & Best & $\mathbf{0}$ & $\mathbf{0}$ & $\mathbf{0}$ & $\mathbf{0}$ & $\mathbf{0}$ & $\mathbf{0}$ \\
\hline$f_{3}$ & Mean & $\mathbf{0}$ & $\mathbf{0}$ & $\mathbf{0}$ & $\mathbf{0}$ & $\mathbf{0}$ & $\mathbf{0}$ \\
\hline & Std & $\mathbf{0}$ & $\mathbf{0}$ & $\mathbf{0}$ & $\mathbf{0}$ & $\mathbf{0}$ & $\mathbf{0}$ \\
\hline & Best & $4.85 \mathrm{E}-004$ & $2.18 \mathrm{E}-003$ & $\mathbf{6 . 2 7 E}-\mathbf{0 0 5}$ & $6.06 \mathrm{E}-004$ & $3.55 \mathrm{E}-004$ & $4.16 \mathrm{E}-004$ \\
\hline$f_{4}$ & Mean & $5.43 \mathrm{E}-003$ & $8.91 \mathrm{E}-003$ & $\mathbf{1 . 1 3 E}-\mathbf{0 0 3}$ & $8.37 \mathrm{E}-003$ & $4.35 \mathrm{E}-003$ & $4.79 \mathrm{E}-003$ \\
\hline & Std & $5.80 \mathrm{E}-003$ & $8.45 \mathrm{E}-003$ & $\mathbf{1 . 5 9 E}-\mathbf{0 0 3}$ & $6.71 \mathrm{E}-003$ & $3.17 \mathrm{E}-003$ & $4.76 \mathrm{E}-003$ \\
\hline & Best & $\mathbf{0}$ & $\mathbf{0}$ & $\mathbf{0}$ & $\mathbf{0}$ & $\mathbf{0}$ & $\mathbf{0}$ \\
\hline$f_{5}$ & Mean & $\mathbf{0}$ & $\mathbf{0}$ & $\mathbf{0}$ & $\mathbf{0}$ & $\mathbf{0}$ & $\mathbf{0}$ \\
\hline & Std & $\mathbf{0}$ & $\mathbf{0}$ & $\mathbf{0}$ & $\mathbf{0}$ & $\mathbf{0}$ & $\mathbf{0}$ \\
\hline & Best & $\mathbf{0}$ & $\mathbf{0}$ & $\mathbf{0}$ & $\mathbf{0}$ & $\mathbf{0}$ & $\mathbf{0}$ \\
\hline$f_{6}$ & Mean & $\mathbf{0}$ & $\mathbf{0}$ & $\mathbf{0}$ & $\mathbf{0}$ & $\mathbf{0}$ & $\mathbf{0}$ \\
\hline & Std & $\mathbf{0}$ & $\mathbf{0}$ & $\mathbf{0}$ & $\mathbf{0}$ & $\mathbf{0}$ & $\mathbf{0}$ \\
\hline
\end{tabular}

From Table 2, an interesting result is that the six WOA-based algorithms are able to find the global optima value $(0)$ on three test functions $\left(f_{3}, f_{5}\right.$, and $\left.f_{6}\right)$ with fixed number of iterations. For test functions $f_{1}$ and $f_{2}$, the mean values obtained by six algorithms are highly near global optimum. With respect to WOA algorithm, SinWOA and TanWOA can obtain better results on two test functions $\left(f_{1}\right.$ and $\left.f_{2}\right)$ and similar results on three test functions $\left(f_{3}, f_{5}\right.$, and $\left.f_{6}\right)$. However, for function $f_{4}$, WOA can find better results. Compare with WOA algorithm, CosWOA, LogWOA, and SquareWOA are able to provide better results for test functions $f_{1}$ and $f_{2}$. For the rest of test functions, four algorithms find similar results.
In general, the performance of the CosWOA algorithm is the best and the standard WOA algorithm is the worst among the six compared algorithms in a statistically significant fashion.

Fig.3 illustrates the convergence curves of fitness values with respect to the number of iterations for the six benchmark test functions with $D=30$. It can be observed from Fig. 3 that the CosWOA algorithm is faster than the standard WOA and the other four improved WOA algorithms on all the benchmark test functions. 


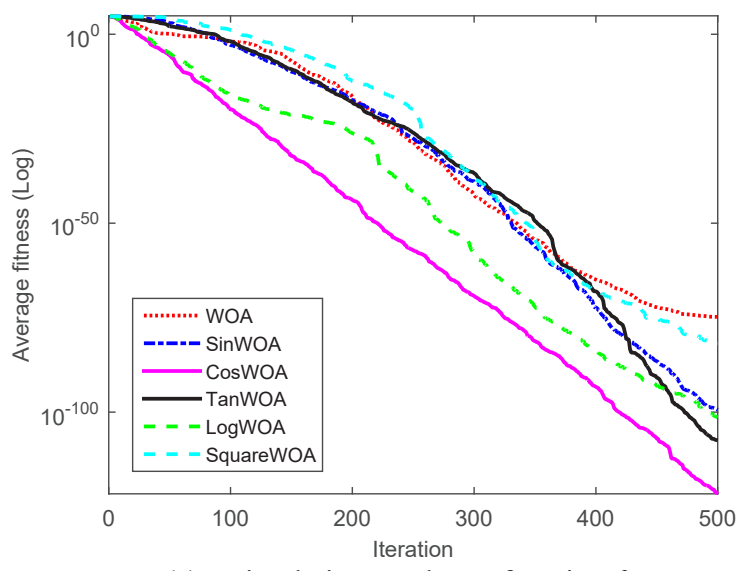

(a) Simulation results on function $f_{1}$

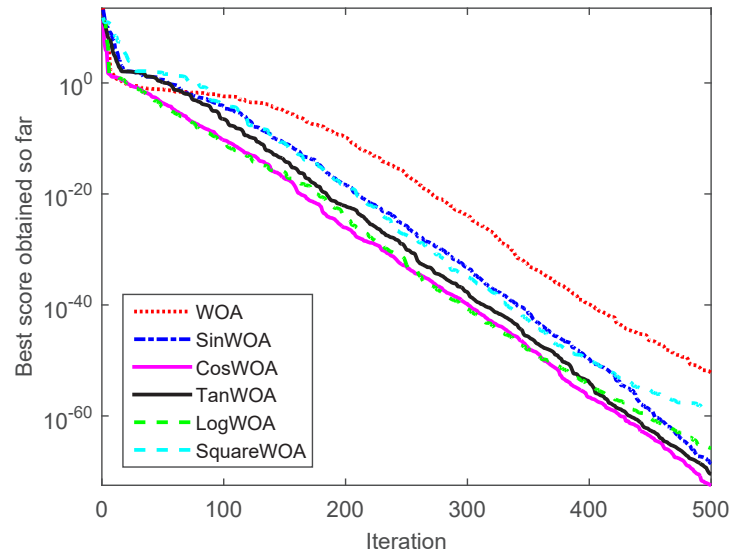

(b) Simulation results on function $f_{2}$

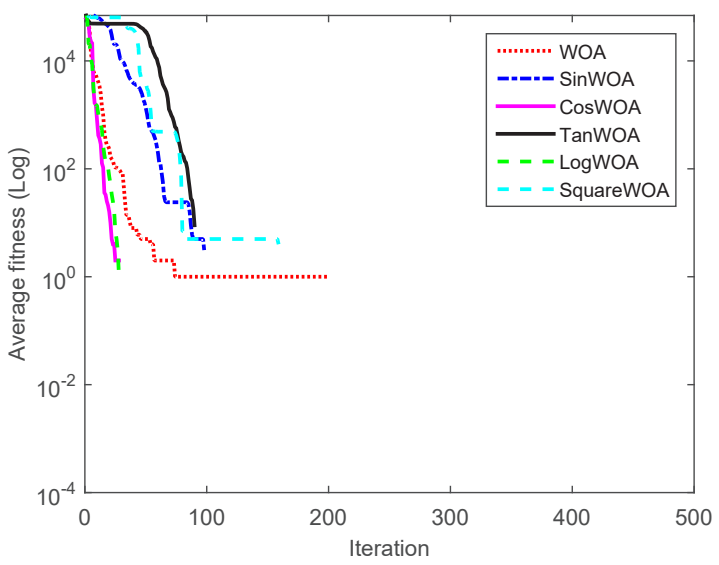

(c) Simulation results on function $f_{3}$

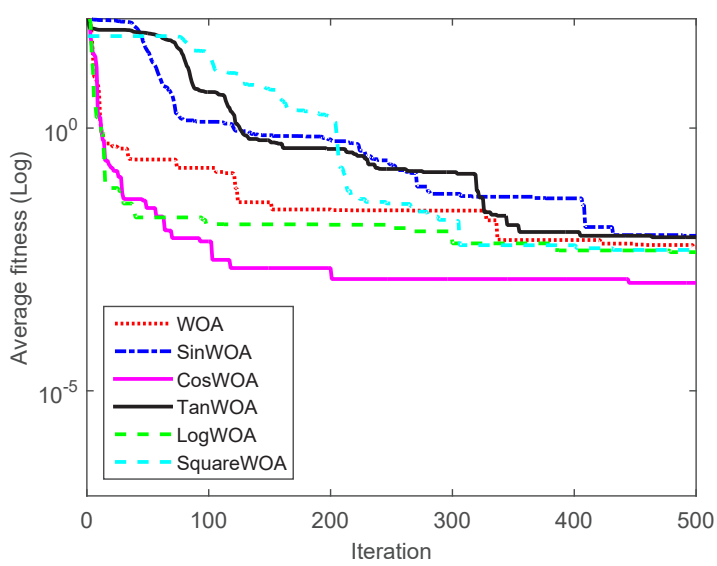

(d) Simulation results on function $f_{4}$

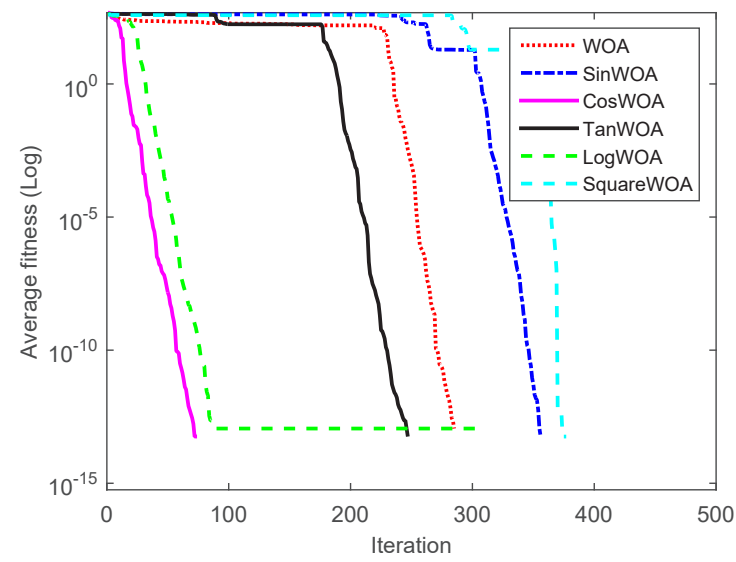

(e) Simulation results on function $f_{5}$

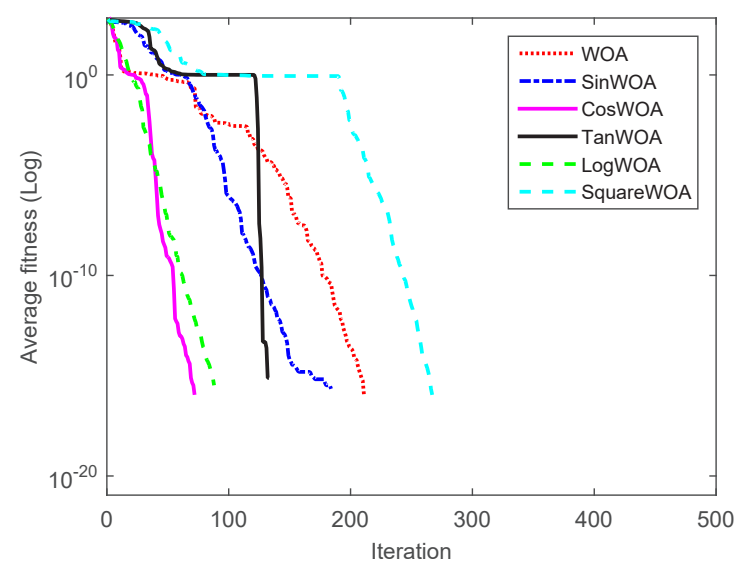

(f) Simulation results on function $f_{6}$

Figure 3. Convergence curves of six functions.

\section{Conclusion}

Whale optimization algorithm (WOA) is a novel metaheuristic optimization technique inspired by the bubblenet hunting behaviour of humpback whales. Exploration and exploitation abilities of WOA are guaranteed by the adaptive values of $a$. This paper introduces nonlinearly distance control parameter strategies to design improved WOA algorithm. Six benchmark test functions were employed to verify the performance of the proposed improved WOA algorithm. The experimental results show that the proposed improved WOA with nonlinearly distance control strategies can provide highly competitive results.

\section{Acknowledgements}

This work is supported in part by the National Natural Science Foundation of China under the Grant Number 61463009, and supported in part by the Science and Technology Foundation of Guizhou Province under the Grant Number [2016]1022.

\section{References}

1. S. Mirjalili, A. Lewis, Advances in Engineering Software, 95, (2016), 51-67. 
2. R.H. Bhesdadiya, P. Jangir, N. Jangir, I.N. Trivedi, D. Ladumor, Indian Journal of Science and Technology, 7, (2016), 1-15.

3. C.H.S. Kumar, R.S. Rao, International Journal of Renewable Energy Development, 5, (2016), 225232.

4. R.H. Bhesdadiya, S.A. Parmar, I.N. Trivedi, P. Jangir, M. Bhoye, N. Jangir, Indian Journal of Science and Technology, 7, (2016), 1-12.

5. M.M. Mafarja, S. Mirjalili, Neurocomputing, 260, (2017), 302-312.

6. D. Oliva, M.A.E. Aziz, A.E. Hassanien, Applied Energy, 200, (2017), 141-154.

7. M.A.E. Aziz, A.A. Ewees, A.E. Hassanien, Expert Systems with Applications, 83, (2017), 242-256.

8. I.N. Trivedi, P. Jangir, N. Jangir, A. Kumar, D. Ladumor, Indian Journal of Science and Technology, 9, (2016), 1-6.

9. Y. Ling, Y.Q. Zhou, Q.F. Luo, IEEE Access, (to be publised)

10. H.P. Hu, Y.P. Bai, T. Xu, International Journal of Circuits, Systems and Signal Processing, 11, (2017), 12-26. 